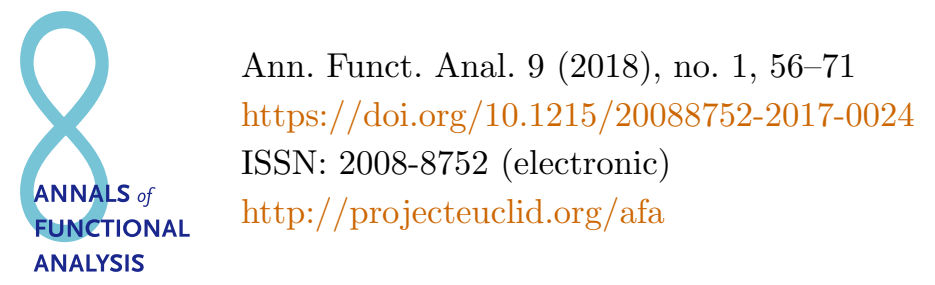

\title{
BASES IN SOME SPACES OF WHITNEY FUNCTIONS
}

\author{
ALEXANDER GONCHAROV* ${ }^{*}$ and ZELIHA URAL \\ Communicated by R. Mortini
}

\begin{abstract}
We construct topological bases in spaces of Whitney functions on Cantor sets, which were introduced by the first author. By means of suitable individual extensions of basis elements, we construct a linear continuous extension operator, when it exists for the corresponding space. In general, elements of the basis are restrictions of polynomials to certain subsets. In the case of small sets, we can present strict polynomial bases as well.
\end{abstract}

\section{INTRODUCTION}

This article is supplementary to [5], where the extension problem is discussed for equilibrium Cantor sets $K(\gamma)$ introduced in [4]. The set $K(\gamma)$ is defined by means of a sequence of parameters $\gamma=\left(\gamma_{s}\right)_{s=0}^{\infty}$ and can be considered as a generalization of the classical quadratic Julia set, but as opposed to Julia sets, it is more flexible with respect to its features.

Following [10] (see also [1] and [2]), we say that a compact set $K \subset \mathbb{R}^{d}$ has the extension property $(\mathrm{EP})$ if, for the space $\mathcal{E}(K)$ of Whitney jets on $K$, there exists a linear continuous extension operator $W: \mathcal{E}(K) \rightarrow C^{\infty}\left(\mathbb{R}^{d}\right)$. In [5], we present a characterization of EP for $K(\gamma)$ and, by means of local Newton interpolations, construct an operator $W$, when it exists. This approach goes back to [8] (see also [9]), so we can say that $W$ is a local version of the Pawlucki-Pleśniak operator. Here, we construct topological bases in the spaces $\mathcal{E}(K(\gamma))$. The construction follows [3]. Besides, for $K(\gamma)$ with EP, we present an extension operator $W$ by individual extensions of basis elements.

Copyright 2018 by the Tusi Mathematical Research Group.

Received Aug. 23, 2016; Accepted Feb. 7, 2017.

First published online Jun. 29, 2017.

${ }^{*}$ Corresponding author.

2010 Mathematics Subject Classification. Primary 46E10; Secondary 46A35, 28 A80.

Keywords. Whitney spaces, extension problem, topological bases. 
The article is organized as follows. Section 2 contains definitions and some auxiliary results about the sets $K(\gamma)$. In Sections 3 and 4, we show that the method from [3] can be adapted to our case as well. In Section 5, by means of extension of basis elements, we construct an extension operator $W$ for the spaces $\mathcal{E}(K(\gamma))$, provided $K(\gamma)$ has EP. In the case when the space has a Faber basis, the operator $W$ coincides with the operator presented in [5].

For a finite set $A \subset \mathbb{R}$, let $\#(A)$ be the cardinality of $A$. Given $x \in \mathbb{R}$, by $d_{k}(x, A)$ we denote distances from $x$ to the points of $A$ arranged in nondecreasing order, so $d_{k}(x, A)=\left|x-a_{m_{k}}\right| \nearrow$. Also, $\lfloor a\rfloor$ is the greatest integer less than or equal to $a$, and $|A|$ is the diameter of $A$.

\section{UNIFORM DISTRIBUTION OF POINTS}

As in [5], we consider $\gamma=\left(\gamma_{s}\right)_{s=1}^{\infty}$ with $0<\gamma_{s} \leq 1 / 32$ and $\sum_{s=1}^{\infty} \gamma_{s}<\infty$. Let $r_{0}=1$, let $P_{2}(x)=x(x-1)$, and let $r_{s}=\gamma_{s} r_{s-1}^{2}, P_{2^{s+1}}=P_{2^{s}}\left(P_{2^{s}}+r_{s}\right)$ where $s \in \mathbb{N}$. Then $E_{s}:=\left\{x \in \mathbb{R}: P_{2^{s+1}}(x) \leq 0\right\}=\bigcup_{j=1}^{2^{s}} I_{j, s}$, where the $s$ th level basic intervals $I_{j, s}$ are disjoint. Since $E_{s+1} \subset E_{s}$, we have a Cantor-type set $K(\gamma):=\bigcap_{s=0}^{\infty} E_{s}$.

For the length $\ell_{j, s}$ of the interval $I_{j, s}$, by Lemma 6 in [4], we have

$$
\delta_{s}<\ell_{j, s}<C_{0} \delta_{s} \quad \text { for } 1 \leq j \leq 2^{s},
$$

where $\delta_{0}:=1, \delta_{s}:=\gamma_{1} \gamma_{2} \cdots \gamma_{s}$ for $s \in \mathbb{N}$ and $C_{0}=\exp \left(16 \sum_{k=1}^{\infty} \gamma_{k}\right)$. Clearly,

$$
r_{k}=\delta_{k} \delta_{k-1} \delta_{k-2}^{2} \delta_{k-3}^{4} \cdots \delta_{0}^{2^{k-1}}
$$

Each $I_{j, s}$ contains two adjacent basic subintervals $I_{2 j-1, s+1}$ and $I_{2 j, s+1}$. Let $h_{j, s}=$ $\ell_{j, s}-\ell_{2 j-1, s+1}-\ell_{2 j, s+1}$ be the distance between them. As in [4], Lemma 4,

$$
h_{j, s} \geq 7 / 8 \cdot \ell_{j, s}>7 / 8 \cdot \delta_{s}
$$

for all $s$ and $1 \leq j \leq 2^{s}$ and

$$
\ell_{2 j-1, s+1}+\ell_{2 j, s+1}<4 \ell_{j, s}
$$

We decompose the zeros of $P_{2^{s}}$ into $s$ groups: $X_{0}=\left\{x_{1}, x_{2}\right\}=\{0,1\}, X_{1}=$ $\left\{x_{3}, x_{4}\right\}=\left\{\ell_{1,1}, 1-\ell_{2,1}\right\}, \ldots, X_{k}=\left\{\ell_{1, k}, \ell_{1, k-1}-\ell_{2, k}, \ldots, 1-\ell_{2^{k}, k}\right\}$ for $k \leq s-1$, so $X_{k}$ contains all zeros of $P_{2^{k+1}}$ that are not zeros of $P_{2^{k}}$. If $Y_{s}=\bigcup_{k=0}^{s} X_{k}$, then $P_{2^{s}}(x)=\prod_{x_{k} \in Y_{s-1}}\left(x-x_{k}\right)$. Clearly, $\#\left(X_{s}\right)=2^{s}$ for $s \in \mathbb{N}$ and $\#\left(Y_{s}\right)=2^{s+1}$ for $s \in \mathbb{Z}_{+}$. The elements of $X_{s}$ are called sth-type points.

We put all points $\left(x_{k}\right)_{k=1}^{\infty}$ from $\bigcup_{k=0}^{\infty} X_{k}$ in order by means of the rule of increase of type. The order of $\left(x_{k}\right)_{k=1}^{4}$ is given above. To put the points from $X_{2}$ in order, we increasingly arrange the points from $Y_{1}$, so $Y_{1}=\left\{x_{1}, x_{3}, x_{4}, x_{2}\right\}$. After this we increase the index of each point by 4 . This gives the ordering $X_{2}=\left\{x_{5}, x_{7}, x_{8}, x_{6}\right\}$. Similarly, indices of increasingly arranged points from $Y_{k-1}=\left\{x_{i_{1}}, x_{i_{2}}, \ldots, x_{i_{2^{k}}}\right\}$ define the ordering $X_{k}=\left\{x_{i_{1}+2^{k}}, x_{i_{2}+2^{k}}, \ldots, x_{i_{2^{k}}+2^{k}}\right\}$. We see that $x_{j+2^{k}}=x_{j} \pm$ $\ell_{m, k}$, where the sign and $m$ are uniquely defined by $j$.

A useful feature of this order is that for each $N$, the points $Z:=\left(x_{k}\right)_{k=1}^{N}$ are distributed uniformly on $K(\gamma)$ in the following sense. Suppose that $2^{n} \leq N<$ 
$2^{n+1}$. Then the binary representation

$$
N=2^{n}+2^{m}+\cdots+2^{i}+\cdots+2^{w} \text { with } 0 \leq w<\cdots<i<\cdots<m<n
$$

generates the decomposition $Z=Z_{n} \cup Z_{m} \cup \cdots \cup Z_{w}$ with $Z_{n}=Y_{n-1}$ and $Z_{m} \cup$ $\cdots \cup Z_{w} \subset X_{n}$. Here, $\#\left(Z_{i}\right)=2^{i}$ for $i \in \mathcal{N}:=\{w, \ldots, m, n\}$, and each basic interval of $i$ th level contains just one point from $Z_{i}$. Also, for each $s$ and $i, j \leq 2^{s}$,

$$
\left|\#\left(Z \cap I_{i, s}\right)-\#\left(Z \cap I_{j, s}\right)\right| \leq 1
$$

In what follows, we will associate with a number $N$ not only the sets $Z$ and $\mathcal{N}$, but also the product $\prod_{i \in \mathcal{N}} r_{i}$, where $r_{i}$ is defined in (2.2). We combine together all the $\delta_{k}$ 's that constitute this product and arrange them in nondecreasing order: $\prod_{i \in \mathcal{N}} r_{i}=\prod_{m=1}^{N} \rho_{m}=\prod_{k=0}^{n} \delta_{k}^{s_{k}(N)}$ with $\sum_{k=0}^{n} s_{k}(N)=N, \rho_{m} \leq \rho_{m+1}$. For example, $N=2^{n}$ gives $\prod_{m=1}^{N} \rho_{m}=r_{n}$, whereas $N=21$ generates $\mathcal{N}=\{0,2,4\}$ and $\prod_{m=1}^{21} \rho_{m}=\delta_{4} \delta_{3} \delta_{2}^{3} \delta_{1}^{5} \delta_{0}^{11}$. For each $N \geq 1$ and $k \geq 0$, the corresponding degrees are given by the formula

$$
s_{k}(N)=\left\lfloor 2^{-k-1}\left(N+2^{k}\right)\right\rfloor .
$$

From here it follows that, for $N+1=2^{m}(2 p+1)$, the values $s_{k}(N)$ and $s_{k}(N+1)$ coincide for all $k$ except $k=m$, where $s_{m}(N+1)=s_{m}(N)+1$. We choose similarly the set $Z=\left(x_{k, j, s}\right)_{k=1}^{N}$ on any $I_{j, s}$. If $2^{n} \leq N<2^{n+1}$, then $Z$ includes $2^{n}$ zeros of $P_{2^{s+n}}$ on $I_{j, s}$ and $N-2^{n}$ points of the type $s+n$. Let $r_{i, s}=\delta_{s+i} \delta_{s+i-1} \delta_{s+i-2}^{2} \cdots \delta_{s}^{2^{i-1}}$ and $\prod_{i \in \mathcal{N}} r_{i, s}=\prod_{m=1}^{N} \rho_{m, s}$. We estimate the sup-norm of $f_{N}(x)=\prod_{k=1}^{N}\left(x-x_{k, j, s}\right)$ on $K(\gamma) \cap I_{j, s}$ in terms of the last product.

Lemma 2.1. In the above notation,

$$
(7 / 8)^{N} \prod_{k=1}^{N} \rho_{k, s} \leq\left|f_{N}\right|_{0, K(\gamma) \cap I_{j, s}} \leq C_{0}^{N} \prod_{k=1}^{N} \rho_{k, s} .
$$

Proof. For brevity, let us consider the interval $I_{j, s}=[0,1]$, since the proof for the general case is the same. Thus, we drop the subscripts $j$ and $s$. For $x \in K(\gamma)$, we have $\left|f_{N}(x)\right|=\prod_{k=1}^{N}\left|x-x_{k}\right|=\prod_{i \in \mathcal{N}} \prod_{x_{k} \in Z_{i}}\left|x-x_{k}\right|$. For each $i \in \mathcal{N}$, we consider the chain of basic intervals containing $x: x \in I_{j_{0}, i} \subset I_{j_{1}, i-1} \subset \cdots \subset I_{j_{i}, 0}=[0,1]$. Since the points from $Z_{i}$ are uniformly distributed, the interval $I_{j_{0}, i}$ contains just one point from $Z_{i}$, as well as $I_{j_{1}, i-1} \backslash I_{j_{0}, i}$. Also, $\#\left(Z \cap\left(I_{j_{k}, i-k} \backslash I_{j_{k-1}, i-k+1}\right)\right)=2^{k-1}$ for $1 \leq k \leq i$. Therefore, $\prod_{x_{k} \in Z_{i}}\left|x-x_{k}\right| \leq \ell_{j_{0}, i} \ell_{j_{1}, i-1} \ell_{j_{2}, i-2}^{2} \cdots \ell_{j_{i}, 0}^{2^{i-1}}<C_{0}^{2^{i}} r_{i}$, by (2.1) and (2.2). From this, $\left|f_{N}(x)\right| \leq C_{0}^{N} \prod_{i \in \mathcal{N}} r_{i}$ and

$$
\left|f_{N}\right|_{0, K(\gamma)} \leq C_{0}^{N} \prod_{k=1}^{N} \rho_{k}
$$

The bound (2.7) is sharp with respect to the product $\prod_{k=1}^{N} \rho_{k}$. Indeed, let us consider $\left|f_{N}\left(x_{N+1}\right)\right|$. As above, $x_{N+1} \in I_{j_{0}, n} \subset I_{j_{1}, n-1} \subset \cdots \subset I_{j_{n}, 0}=[0,1]$. Hence, $\prod_{x_{k} \in Z_{n}}\left|x_{N+1}-x_{k}\right| \geq \ell_{j_{0}, n} h_{j_{1}, n-1} h_{j_{2}, n-2}^{2} \cdots h_{j_{n}, 0}^{2^{n-1}}>(7 / 8)^{2^{n}} r_{n}$, by (2.3).

As for $\prod_{x_{k} \in Z_{m}}\left|x_{N+1}-x_{k}\right|$, we observe that the point $x_{N+1}$ must be in some interval $I_{j, m+1}$ which is free of points from $A:=Z_{m} \cup \cdots \cup Z_{w}$. Indeed, the set 
$\left\{x_{N+1}\right\} \cup A$ contains at most $2^{m+1}$ points that are uniformly distributed, so each $I_{j, m+1}$ contains at most one point from this set. Thus, $x_{N+1}$ and its closest point from $Z_{m}$ are located in distinct intervals of the $(m+1)$ th level. Arguing as above, we see that $\prod_{x_{k} \in Z_{m}}\left|x_{N+1}-x_{k}\right| \geq h_{j_{0}, m} h_{j_{1}, m-1} h_{j_{2}, m-2}^{2} \cdots h_{j_{m}, 0}^{2^{m-1}}>(7 / 8)^{2^{m}} r_{m}$.

In a similar fashion, $\prod_{x_{k} \in Z_{i}}\left|x_{N+1}-x_{k}\right|>(7 / 8)^{2^{i}} r_{i}$ for each $i \in \mathcal{N}$. Therefore,

$$
\left|f_{N}\right|_{0, K(\gamma)} \geq\left|f_{N}\left(x_{N+1}\right)\right| \geq(7 / 8)^{N} \prod_{k=1}^{N} \rho_{k} .
$$

Remark. Given $x \in K(\gamma)$, we have $\left|f_{N}(x)\right|=\prod_{k=1}^{N} d_{k}(x, Z)$. The lengths of basic intervals of the same level may be rather different (we can say only that $\ell_{j, s}<C_{0} \ell_{i, s}$, by (2.1)). For this reason, as $k$ increases and $x, y$ belong to different parts of $K(\gamma)$, the values $d_{k}(x, Z)$ and $d_{k}(y, Z)$ may increase in quite different fashions. Nevertheless, the product $\prod_{k=1}^{N} \rho_{k}$ is defined by $N$ only, so it does not depend on the choice of $x$.

In the following technical lemmas, we use the decomposition (2.5). Let $2^{n} \leq$ $N<2^{n+1}$ and a basic interval $I=I_{j, s}$ be given. Suppose that $Z=\left(x_{k}\right)_{k=1}^{N}$ and $\tilde{Z}=\left(x_{k}\right)_{k=1}^{N+1}$ are chosen on $I$ by the rule of increase of type. Write $C_{1}=$ $8 / 7 \cdot\left(C_{0}+1\right)$.

Lemma 2.2. For each $x \in \mathbb{R}$ with $\left.\operatorname{dist}\left(x, K(\gamma) \cap I_{j, s}\right)\right) \leq \delta_{s+n}$ and $z \in \tilde{Z}$, we have $\delta_{s+n} \prod_{k=2}^{N} d_{k}(x, Z) \leq C_{1}^{N} \prod_{k=2}^{N+1} d_{k}(z, \tilde{Z})$.

Proof. As above, for brevity, we take $s=0, j=1$. Let $\tilde{x} \in K(\gamma)$ realize the distance above. Also, let $x_{p} \in Z_{p} \subset Z$ be such that $d_{1}(x, Z)=\left|x-x_{p}\right|$. Of course, $x_{p}$ may coincide with $\tilde{x}$. Clearly,

$$
\delta_{n} \prod_{k=2}^{N} d_{k}(x, Z)=\prod_{i \in \mathcal{N}, i \neq p} \prod_{k=1}^{2^{i}} d_{k}\left(x, Z_{i}\right) \cdot\left(\delta_{n} \prod_{k=2}^{2^{p}} d_{k}\left(x, Z_{p}\right)\right) .
$$

For $i \neq p$, let $\tilde{x} \in I_{j_{0}, i} \subset I_{j_{1}, i-1} \subset \cdots \subset I_{j_{i}, 0}=I$. As in Lemma 2.1, for fixed $q$ with $1 \leq q \leq i$ we consider $2^{q-1}$ points $x_{k}$ from the set $I_{j_{q}, i-q} \backslash I_{j_{q-1}, i-q+1}$. For each of them we have $\left|x-x_{k}\right| \leq|x-\tilde{x}|+\ell_{j_{q}, i-q} \leq\left(C_{0}+1\right) \delta_{i-q}$. A similar estimation is valid for $x_{k} \in I_{j_{0}, i}$. Combining these gives $\prod_{x_{k} \in Z_{i}}\left|x-x_{k}\right| \leq\left(C_{0}+1\right)^{2^{i}} r_{i}$. The terms $d_{k}\left(x, Z_{p}\right)$ for $2 \leq k \leq 2^{p}$ can be handled in much the same way: $\prod_{k=2}^{2^{p}} d_{k}\left(x, Z_{p}\right) \leq\left(C_{0}+1\right)^{2^{p}-1} \frac{r_{p}}{\delta_{p}}$. This yields $\delta_{n} \prod_{k=2}^{N} d_{k}(x, Z) \leq\left(C_{0}+1\right)^{N} \prod_{i \in \mathcal{N}} r_{i}$, as $\delta_{n} \leq \delta_{p}$.

It is sufficient to show that

$$
\prod_{k=2}^{N+1} d_{k}(z, \tilde{Z}) \geq(7 / 8)^{N} \prod_{i \in \mathcal{N}} r_{i}
$$

The case $N+1=2^{n+1}$ follows immediately by the argument of Lemma 2.1. Suppose that $N+1<2^{n+1}$. First consider $w=0$ in (2.5), so $N=2^{n}+2^{m}+$ $\cdots+2^{u}+2^{v-1}+2^{v-2}+\cdots+2+1$ with some $1 \leq v<u$ and, correspondingly, $N+1=2^{n}+\cdots+2^{u}+2^{v}$. Fix $z \in \tilde{Z}$ and the chain $z \in I_{j_{0}, n} \subset I_{j_{1}, n-1} \subset \cdots \subset I_{j_{n}, 0}$. 
Here, $z$ is an endpoint of $I_{j_{0}, n}$. Suppose that $z \in Z_{n}$ and that another endpoint of $I_{j_{0}, n}$ is $z_{p} \in Z_{p} \subset \tilde{Z}$. We estimate separately $\prod_{x_{k} \in Z_{q}}\left|z-x_{k}\right|$ for each $q$ from the binary decomposition of $N+1$.

For $q=n$ we have, as in Lemma 2.1, $d_{1}\left(z, Z_{n}\right)=\ell_{j_{1}, n-1}, d_{2}\left(z, Z_{n}\right) \geq h_{j_{2}, n-2}, \ldots$, and $\prod_{x_{k} \in Z_{n}}\left|z-x_{k}\right|>(7 / 8)^{2^{n}-2} r_{n} / \delta_{n}$.

If $p<q \leq m$, then $\prod_{x_{k} \in Z_{q}}\left|z-x_{k}\right| \geq(7 / 8)^{2^{q}} r_{q}$. Indeed, $z_{p} \in I_{j_{n-q-1}, q+1}$, which may contain at most one point from the set $Z_{q} \cup \cdots \cup Z_{p} \cup \cdots \cup Z_{v}$. Hence, the point $x_{k} \in Z_{q}$ which is closest to $z$ belongs to the adjacent interval of the $(q+1)$ th level and $d_{1}\left(z, Z_{q}\right) \geq h_{j_{n-q}, q}$. Continuing in this fashion, by (2.3), we get the desired bound for given $q$.

We now handle the case $q=p$. Here, $d_{1}\left(z, Z_{p}\right)=\left|z-z_{p}\right|=\ell_{j_{0}, n}>\delta_{n}$. Since $z_{p} \in$ $I_{j_{n-p}, p}$, this interval cannot contain another point from $Z_{p}$. Therefore, $d_{2}\left(z, Z_{p}\right) \geq$ $h_{j_{n-p+1}, p-1} \geq 7 / 8 \delta_{p-1}$ and $\prod_{x_{k} \in Z_{p}}\left|z-x_{k}\right| \geq(7 / 8)^{2^{p}} r_{p} \delta_{n} / \delta_{p}$.

To deal with indices $q<p$, let us take the nearest to $z$ point $z_{p_{1}}$ from the set $\tilde{Z} \backslash\left(Z_{n} \cup Z_{m} \cup \cdots \cup Z_{p}\right)=Z_{t} \cup \cdots \cup Z_{p_{1}} \cup \cdots \cup Z_{v}$. If $p_{1}<q \leq t$, then, as above, $\prod_{x_{k} \in Z_{q}}\left|z-x_{k}\right| \geq(7 / 8)^{2^{q}} r_{q}$. If $q=p_{1}$, then $\prod_{x_{k} \in Z_{p_{1}}}\left|z-x_{k}\right| \geq(7 / 8)^{2^{p_{1}}} r_{p_{1}} \delta_{p} / \delta_{p_{1}}$. Indeed, the interval $I_{j_{n-p-1}, p+1}$ contains $z_{p}$, so it cannot contain another point from $Z_{p} \cup \cdots \cup Z_{p_{1}} \cup \cdots \cup Z_{v}$. Therefore, $z$ and $z_{p_{1}}$ must be in different intervals of the $(p+1)$ th level. Then $d_{1}\left(z, Z_{p_{1}}\right) \geq h_{j_{n-p}, p}$. Now $I_{j_{n-p_{1}}, p_{1}}$ contains $z_{p_{1}}$, so $d_{2}\left(z, Z_{p_{1}}\right) \geq h_{j_{n-p_{1}+1}, p_{1}-1}$. The values $d_{k}\left(z, Z_{p_{1}}\right)$ for $k>2$ we estimate in much the same way as in the case $q=p$.

We continue in this way and combine all bounds up to $p_{k}=v$ together:

$$
\begin{aligned}
(8 / 7)^{N} \cdot \prod_{k=2}^{N+1} d_{k}(z, \tilde{Z}) & \geq \frac{r_{n}}{\delta_{n}} r_{m} \cdots r_{p} \frac{\delta_{n}}{\delta_{p}} r_{t} \cdots r_{p_{1}} \frac{\delta_{p}}{\delta_{p_{1}}} \cdots r_{p_{2}} \frac{\delta_{p_{1}}}{\delta_{p_{2}}} \cdots r_{p_{k}} \frac{\delta_{p_{k-1}}}{\delta_{p_{k}}} \\
& =r_{n} \cdots r_{u} r_{v} / \delta_{v}=\prod_{i \in \mathcal{N}} r_{i}=r_{n} \cdots r_{u} r_{v-1} \cdots r_{1} r_{0},
\end{aligned}
$$

since $r_{v-1} r_{v-2} \cdots r_{1} r_{0}=r_{v} / \delta_{v}$, as is easy to check. This yields (2.9). The cases $z \in Z_{p} \subset \tilde{Z}$ and $w>0$ are very similar.

In the next lemma we consider the same $N$ and $\tilde{Z}$ as above, but we now arrange the points in increasing order. Thus, $\tilde{Z}=\left(z_{k}\right)_{k=1}^{N+1} \subset I$ with $z_{k} \nearrow$. For $q=2^{m}-1$ with $m<n$ and $1 \leq j \leq N+1-q$, let $J=\left\{z_{j}, \ldots, z_{j+q}\right\}$ consist of $2^{m}$ consecutive points from $\tilde{Z}$. Given $j$, we consider all possible chains of strict inclusions of segments of natural numbers:

$$
[j, j+q]=\left[a_{0}, b_{0}\right] \subset\left[a_{1}, b_{1}\right] \subset \cdots \subset\left[a_{N-q}, b_{N-q}\right]=[1, N+1],
$$

where $a_{k}=a_{k-1}, b_{k}=b_{k-1}+1$ or $a_{k}=a_{k-1}-1, b_{k}=b_{k-1}$ for $1 \leq k \leq N-q$. Every chain generates the product $\prod_{k=1}^{N-q}\left(z_{b_{k}}-z_{a_{k}}\right)$. For fixed $J$, let $\Pi(J)$ denote the minimum of such products for all possible chains.

Lemma 2.3. For each $J \subset \tilde{Z}$, there is $\tilde{z} \in J$ such that $\prod_{k=q+2}^{N+1} d_{k}(\tilde{z}, \tilde{Z}) \leq \Pi(J)$. 
Proof. We take $I=[0,1]$, as the corresponding change of indices gives the proof in the general case. Fix $J \subset \tilde{Z}$. Let $J_{k}=\left[z_{a_{k}}, z_{b_{k}}\right]$. Then (2.10), which defines $\Pi(J)$, generates inclusions $J \subset J_{1} \subset \cdots \subset J_{N-q}=I$ with $\Pi(J)=\prod_{k=1}^{N-q}\left|J_{k}\right|$.

Clearly, $\#(\tilde{Z} \backslash J)=N-q$ and each $z \in \tilde{Z} \backslash J$ appears as an endpoint of some $J_{k}$. Let $w_{k}$ be the endpoint of $J_{k}$ in its first appearance. This gives an enumeration of $\tilde{Z} \backslash J$. We aim to find $\tilde{z} \in J$ and a permutation $\left(w_{i_{k}}\right)_{k=1}^{N-q}$ such that for $1 \leq k \leq N-q$ we have $d_{q+1+k}(\tilde{z}, \tilde{Z}) \leq\left|J_{i_{k}}\right|$. Multiplying these inequalities yields the result. Given $\tilde{z}$, let $d_{k}$ be shorthand for $d_{k}(\tilde{z}, \tilde{Z})$.

Recall that $2^{n}+1 \leq \#(\tilde{Z}) \leq 2^{n+1}, Y_{n-1} \subset \tilde{Z} \subset Y_{n}$, and $\#(J)=2^{m}$. The points from $\tilde{Z}$ are distributed uniformly on $I$. Hence, for each basic interval of the $(n-m+1)$ th level, we have

$$
2^{m-1} \leq \#\left(\tilde{Z} \cap I_{j, n-m+1}\right) \leq 2^{m} .
$$

We observe that $J$ may be located on $v$ consecutive intervals of this level with $1 \leq v \leq 3$. Indeed, if $J \subset I_{1} \cup I_{2} \cup I_{3} \cup I_{4}$ with $J \cap I_{k} \neq \emptyset$, then all points from $\tilde{Z}$ in

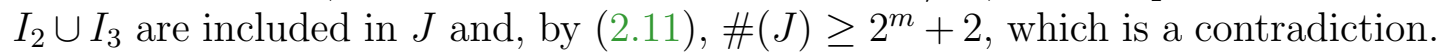
Let us consider all possible values of $v$.

(1) Let $J \subset I_{1}:=I_{j, n-m+1}$ for some $j$. Here, $J=\tilde{Z} \cap I_{1}$ and any point $z \in J$ may serve as $\tilde{z}$, since distances $d_{k}$ for $1 \leq k \leq 2^{m}$ are implemented on certain points of the set $I_{1}$. If $q+2 \leq k \leq N+1$, then $d_{k}$ is $\left|\tilde{z}-w_{i_{k}}\right|$ for some $w_{i_{k}} \in \tilde{Z} \backslash J$ and $d_{k}<\left|J_{i_{k}}\right|$, since $\tilde{z} \in J_{i_{k}}$ and $w_{i_{k}}$ is an endpoint of this interval.

(2) Let $J \subset I_{1} \cup I_{2}$. Suppose first that these intervals are adjacent, that is, $I_{1}=I_{2 j-1, n-m+1}$ and $I_{2}=I_{2 j, n-m+1}$. Let $p:=\#\left(J \cap I_{1}\right)$; that is, $z_{j}, \ldots, z_{j+p-1}$ belong to $I_{1}$, whereas $z_{j+p}, \ldots, z_{j+q} \in I_{2}$. Suppose, for definiteness, that $p \leq$ $2^{m-1}$; that is, at least a half of $J$ is in the right interval. The right endpoint of $I_{2}$ belongs to $Y_{n-1}$, so it is $z_{j+r}$ for some $r$ with $r \geq q$. Thus, $\#\left(\tilde{Z} \cap I_{2}\right)=$ $r-p+1$, where $r-q$ of these points are from $\tilde{Z} \backslash J$. We take $\tilde{z}=z_{j+p}$, the left endpoint of $I_{2}$. Then $d_{k}=z_{j+p+k-1}-\tilde{z}$ for $1 \leq k \leq r-p+1$, since the lengths of basic intervals are smaller than the gaps between them. In particular, $d_{r-p+1}=z_{j+r}-\tilde{z}=\left|I_{2}\right|$. The next distances will be realized on the points from $I_{1}: d_{r-p+2}=\tilde{z}-z_{j+p-1}, \ldots, d_{r+1}=\tilde{z}-z_{j}$. Since $\#\left(\tilde{Z} \cap I_{2}\right) \leq 2^{m} \leq r+1$, the value $d_{2^{m}}$ is $\tilde{z}-z_{j+i}$ for some $i$ with $j \leq i \leq j+p-1$.

Now, for $d_{k}$ with $q+1 \leq k \leq r+1$ we take $w_{i_{k}}=z_{j+k-1}$ on $I_{2}$. Then $\left|J_{i_{k}}\right|>$ $z_{j+k-1}-z_{j}>d_{k}=\tilde{z}-z_{j+r-k+1}$, as $z_{j+k-1}>\tilde{z}$ and $z_{j+r-k+1} \geq z_{j}$. Note that the next values of $d_{k}$ (for $r+2 \leq k \leq N+1$ ) will be implemented on certain points of the set $w_{i_{k}} \in \tilde{Z} \backslash J$. As in the first case, $d_{k}<\left|J_{i_{k}}\right|$.

The same reasoning applies to the case of nonadjacent intervals. Let $I_{1}=$ $I_{2 j-2, n-m+1}$ and $I_{2}=I_{2 j-1, n-m+1} \subset I_{j, n-m}$. Then we take $\tilde{z}$ as the endpoint of an interval containing at least half of $J$, let it be again $I_{2}$. Here, $p$ points from $I_{1} \cap J$ realize some $d_{M+1}, \ldots, d_{M+p}$ and we put into correspondence to them the first $p$ points from $I_{j, n-m} \backslash J$. All other $d_{k}$ 's are realized on some $w_{i_{k}}$ with $d_{k}<\left|J_{i_{k}}\right|$.

(3) Let $J \subset I_{1} \cup I_{2} \cup I_{3}$. Here, $I_{2}$ is completely filled with points of $J$. One of the endpoints of $I_{2}$ belongs to $Y_{n-m-1}$. We take this point as $\tilde{z}$, which, analogously to the previous cases, satisfies the desired condition. 
Lemma 2.4. Let $2^{n} \leq N<2^{n+1}$ and let $Z=\left(x_{k, j, s}\right)_{k=1}^{N}$ be chosen in $I_{j, s}$ by the rule of increase of type. Suppose that $x, y \in I_{i, s+n-q} \subset I_{j, s}$ for some $q<n$ (in general, $x, y \notin K(\gamma))$. Then $\prod_{k=m+1}^{N} \frac{d_{k}(y, Z)}{d_{k}(x, Z)} \leq \exp \left(2^{q}\right)$, where $m=\#\left(I_{i, s+n-q} \cap Z\right)$.

Proof. For brevity, let $I_{j, s}=[0,1], Z=\left(x_{k}\right)_{k=1}^{N}$. Fix $q<n$, any $I_{i, n-q}$, and $x, y$ in this interval. Recall that $I_{i, n-q}$ may contain from $2^{q}$ to $2^{q+1}$ points of $Z$. The distances $d_{k}(y, Z)$ and $d_{k}(x, Z)$ for $1 \leq k \leq m$ are realized on points from $Z \cap I_{i, n-q}$, so we can consider $\left|y-x_{p}\right| /\left|x-x_{p}\right|$ for $x_{p} \in Z \backslash I_{i, n-q}$ only. Let $I_{i, n-q} \subset I_{i_{1}, n-q-1} \subset \cdots \subset I_{i_{n-q}, 0}$. If $x_{p} \in I_{i_{1}, n-q-1} \backslash I_{i, n-q}$, then $\left|y-x_{p}\right| \leq \ell_{i_{1}, n-q-1}$, $\left|x-x_{p}\right| \geq h_{i_{1}, n-q-1}$, and $\left|y-x_{p}\right| /\left|x-x_{p}\right| \leq 8 / 7$, by (2.3). There are at most $2^{q+1}$ such points $x_{p}$. They contribute $(8 / 7)^{2^{q+1}}$ into the common product. For the next step, when $x_{p} \in I_{i_{2}, n-q-2} \backslash I_{i+1, n-q-1}$, we have $\left|y-x_{p}\right| \leq\left|x-x_{p}\right|+\ell_{i, n-q}$ with $\left|x-x_{p}\right| \geq h_{i_{2}, n-q-2} \geq 7 / 8 l_{i_{2}, n-q-2}$. Here, $\left|y-x_{p}\right| /\left|x-x_{p}\right| \leq 1+8 / 7 \cdot 4^{-2}$, by (2.4). Similarly, we get at most $2^{q+k}$ terms in the general product, each of them bounded above by $1+8 / 7 \cdot 4^{-k}$. Here, $2 \leq k \leq n-q$. Thus, $\prod_{k=m+1}^{N} \frac{d_{k}(y, Z)}{d_{k}(x, Z)} \leq$ $(8 / 7)^{2^{q+1}} \prod_{k=2}^{n-q}\left(1+8 / 7 \cdot 4^{-k}\right)^{2^{q+k}}$, which does not exceed $\exp \left(2^{q}\right)$.

As in [5], we use $B_{k}=2^{-k-1} \cdot \log \frac{1}{\delta_{k}}$. By Theorem 5.3 in [5], $K(\gamma)$ has the extension property if and only if $B_{n+s} / \sum_{k=s}^{n+s} B_{k} \rightarrow 0$ as $n \rightarrow \infty$ uniformly with respect to $s$. This condition can be written as

$$
\forall M \exists m, k_{0}: \quad M \cdot B_{k} \leq B_{k-m}+\cdots+B_{k} \quad \text { for } k \geq k_{0}
$$

Here we will use a stronger one

$$
\forall M, Q \exists m, k_{0}: \quad Q+M \cdot B_{k} \leq B_{k-m}+\cdots+B_{k} \quad \text { for } k \geq k_{0},
$$

which will provide continuity of the extension operator constructed by interpolation of functions on the whole set.

When constructing a Faber basis in the space $\mathcal{E}(K(\gamma))$, we also use the clause

$$
\forall Q \exists m, k_{0}: \quad Q \leq B_{k-m}+\cdots+B_{k} \quad \text { for } k \geq k_{0} .
$$

These conditions have "geometric" forms in terms of $\left(\delta_{k}\right)$. For example, (2.13) is

$$
\forall M, Q \exists m, k_{0}: \quad Q^{2^{k}} \cdot \delta_{k-m}^{2^{m}} \cdots \delta_{k-1}^{2} \cdot \delta_{k} \leq \delta_{k}^{M} \quad \text { for } k \geq k_{0}
$$

Let us illustrate the difference between conditions (2.12)-(2.14) for the case of a monotone sequence $\left(B_{k}\right)_{k=1}^{\infty}$. Since only $\gamma_{s} \leq 1 / 32$ are allowed here, we have $B_{k} \geq \log 32 \cdot k 2^{-k-1}$. On the other hand, the values of $B_{k}$ may be as large as we wish for small sets $K(\gamma)$. Condition (2.12) is valid if $B_{k} \searrow, B_{k} \nearrow B<\infty$, or $B_{k} \nearrow \infty$, but slowly, with subexponential growth (i.e., $k^{-1} \log B_{k} \rightarrow 0$ ), by Theorem 7.1 in [5]. In turn, we have (2.13) for $B_{k} \searrow B>0, B_{k} \nearrow B<\infty$, or $B_{k} \nearrow \infty$ of subexponential growth, whereas (2.14) is satisfied with $B_{k} \searrow B>0$ and any $B_{k} \nearrow$. Thus, the sequence of constants $B_{k}$ satisfies all three conditions. 


\section{FABer Bases in the SPACE $\mathcal{E}(K(\gamma))$}

We equip the Whitney space $\mathcal{E}(K(\gamma))$ with the norms

$$
\begin{aligned}
\|f\|_{q}= & |f|_{q, K(\gamma)} \\
& +\sup \left\{\left|\left(R_{y}^{q} f\right)^{(k)}(x)\right| \cdot|x-y|^{k-q}: x, y \in K(\gamma), x \neq y, k=0,1, \ldots, q\right\}
\end{aligned}
$$

for $q \in \mathbb{Z}_{+}$, where $|f|_{q, K(\gamma)}=\sup \left\{\left|f^{(k)}(x)\right|: x \in K(\gamma), k \leq q\right\}$ and $R_{y}^{q} f(x)=$ $f(x)-T_{y}^{q} f(x)$ is the Taylor remainder. By the open mapping theorem, for any $q$ there exist $r \in \mathbb{N}, C>0$ such that

$$
\|f\|_{q} \leq C\|f\|_{r}
$$

for any $f \in \mathcal{E}(K)$. Here, $\left.\left|\|f\|_{q}=\inf \right| F\right|_{q,[0,1]}$, where the infimum is taken over all possible extensions of $f$ to $F \in C^{\infty}[0,1]$ (see [5] for more details; for more insight into the theory of Whitney spaces, see [12] and [6]).

Here we adjust the construction from [3], where the case of symmetric Cantor sets was considered. Let $e_{0} \equiv 1$ and $e_{N}(x)=\prod_{1}^{N}\left(x-x_{k}\right)$ for $N \in \mathbb{N}$, where the points $\left(x_{k}\right)_{1}^{\infty}$ are chosen in $K(\gamma)$ by the rule of increase of type. The divided differences define linear continuous functionals $\xi_{N}(f)=\left[x_{1}, x_{2}, \ldots, x_{N+1}\right] f$ on $\mathcal{E}(K(\gamma))$. By standard properties of divided differences, the system $\left(e_{N}, \xi_{N}\right)_{N=0}^{\infty}$ is biorthogonal and the functionals $\left(\xi_{N}\right)_{N=0}^{\infty}$ are total on $\mathcal{E}(K(\gamma))$; that is, whenever $\xi_{N}(f)=0$ for all $N$, then $f=0$. We show that $\left(e_{N}\right)_{N=0}^{\infty}$ is a topological basis in the space $\mathcal{E}(K(\gamma))$ provided the set $K(\gamma)$ is sufficiently small. Thus, for small sets $K(\gamma)$, the space $\mathcal{E}(K(\gamma))$ possesses a strict polynomial basis. Recall that a polynomial topological basis $\left(P_{n}\right)_{n=0}^{\infty}$ in a functional space is called a Faber (or strict polynomial) basis if $\operatorname{deg} P_{n}=n$ for all $n$. Due to a classical result of Faber, the space $C[a, b]$ does not have such a basis.

Lemma 3.1. Let $p=2^{u}<N / 2$. Then $\left\|e_{N}\right\|_{p} \leq C \cdot C_{0}^{N} N^{p} \cdot \prod_{k=2 p+1}^{N} \rho_{k}$, where $C$ does not depend on $N$ and $\prod_{k=1}^{N} \rho_{k}$ is the product generated by $N$.

Proof. By Lemma 2.1, for $I_{j, s}=[0,1]$, we have $\left|e_{N}\right|_{q, K(\gamma)} \leq C_{0}^{N} \prod_{k=1}^{N} \rho_{k}$ for $q=0$. Our first goal is to generalize it to $q<N$. Let us show that

$$
\left|e_{N}\right|_{q, K(\gamma)} \leq C_{0}^{N-q} N^{q} \prod_{k=q+1}^{N} \rho_{k} .
$$

Fix $x$. Then $\left|e_{N}(x)\right|=\prod_{k=1}^{N} d_{k}(x, Z)$ and the $q$ th derivative of $e_{N}$ at $x$ is the sum of $\frac{N !}{(N-q) !}$ products, where each product contains $N-q$ terms of the form $\left(x-x_{k}\right)$. Hence, $\left|e_{N}^{(q)}(x)\right| \leq N^{q} \prod_{k=q+1}^{N} d_{k}(x, Z)$. Here, for each $k$, we take the smallest $m=m(k)$ with $d_{k}(x, Z) \leq \ell_{j_{m}, i-m}<C_{0} \delta_{i-m}$. By the remark after Lemma 2.1, $\delta_{i-m} \leq \rho_{k}$. The last inequality may be strict if we take $x$ in a part of the set with a high density of points $x_{k}$, for example, near the origin. To deal with $\left\|e_{N}\right\|_{p}$, let us fix $i \leq p$ and $x \neq y$ in $K(\gamma)$. For brevity, let $R:=\left(R_{x}^{p} e_{N}\right)^{(i)}(y)$. We consider two cases: (a) $x, y$ belong to the same interval or (b) two different intervals of the level $n-u$. 
In the first case, let $x, y \in I_{j, n-u}$. By the Lagrange form for the Taylor remainder, we have $|R| \cdot|x-y|^{i-p} \leq\left|e_{N}^{(p)}(\theta)\right|+\left|e_{N}^{(p)}(x)\right|$ for some $\theta \in I_{j, n-u}$. Let $m:=\#\left(I_{j, n-u} \cap Z\right)$. Since the points from $Z$ are distributed uniformly on $K(\gamma)$, we have $p \leq m \leq 2 p$. Hence, $\left|e_{N}^{(p)}(\theta)\right| \leq N^{p} \cdot \prod_{k=p+1}^{N} d_{k}(\theta, Z) \leq N^{p} \cdot \prod_{k=m+1}^{N} d_{k}(\theta, Z)$, as all distances here do not exceed 1. By Lemma 2.4 and the argument in the proof of (3.2), $\left|e_{N}^{(p)}(\theta)\right| \leq e^{p} N^{p} \cdot \prod_{k=m+1}^{N} d_{k}(x, Z) \leq e^{p} N^{p} C_{0}^{N-m} \cdot \prod_{k=m+1}^{N} \rho_{k}$. On the other hand, $\left|e_{N}^{(p)}(x)\right| \leq N^{p} C_{0}^{N-p} \cdot \prod_{k=p+1}^{N} \rho_{k}$. Thus, in the first case, $|R| \cdot|x-y|^{i-p} \leq 2 e^{p} N^{p} C_{0}^{N} \cdot \prod_{k=2 p+1}^{N} \rho_{k}$, since the last product dominates the products of $\rho_{k}$ involved in the estimation of both terms.

In the second case, let $y \notin I_{j, n-u}$, so $|x-y| \geq h_{j_{1}, n-u-1}$, where $x \in I_{j, n-u} \subset$ $I_{j_{1}, n-u-1}$. By $(2.3),|x-y|>7 / 8 \cdot \delta_{n-u-1}$. Now,

$$
|R| \cdot|x-y|^{i-p} \leq\left|e_{N}^{(i)}(y)\right| \cdot|x-y|^{i-p}+\sum_{k=i}^{p}\left|e_{N}^{(k)}(x)\right| \cdot \frac{|x-y|^{k-p}}{(k-i) !}
$$

By (3.2), $\left|e_{N}^{(k)}(x)\right| \cdot|x-y|^{k-p} \leq C_{0}^{N-k} N^{k} \prod_{m=k+1}^{N} \rho_{m} \cdot(8 / 7)^{p-k} \delta_{n-u-1}^{k-p}$. Recall that $I_{j, n-u}$ contains at least $p$ points from $Z$. Therefore, $\rho_{k+1} \cdots \rho_{p} \leq \delta_{n-u-1}^{p-k}$ and $\left|e_{N}^{(k)}(x)\right| \cdot|x-y|^{k-p} \leq(8 / 7)^{p} C_{0}^{N} N^{k} \prod_{m=p+1}^{N} \rho_{m}$. Clearly, $N^{i}+\sum_{k=i}^{p} \frac{N^{k}}{(k-i) !}<N^{p} e$. Combining these yields $|R| \cdot|x-y|^{i-p} \leq(8 / 7)^{p} C_{0}^{N} N^{p} \prod_{m=p+1}^{N} \rho_{m}$. The result follows from a comparison of the estimates in both cases.

We proceed to estimate $\left|\xi_{N}(f)\right|$ for $f \in \mathcal{E}(K(\gamma)), \xi_{N}(f)=\left[z_{1}, \ldots, z_{N+1}\right] f$. As in Lemma 2.3, $\tilde{Z}=\left(z_{k}\right)_{k=1}^{N+1}$ is the set $\left(x_{k}\right)_{k=1}^{N+1}$ arranged in increasing order. Here, $N$ generates the product $\prod_{k=1}^{N} \rho_{k}=\prod_{k=0}^{n} \delta_{k}^{s_{k}(N)}$, whereas for $N+1$ we have $\prod_{k=1}^{N+1} \tilde{\rho}_{k}=\prod_{k=0} \delta_{k}^{s_{k}(N+1)}$ with $s_{k}(N)=s_{k}(N+1)$ for all $k$ except one value, which is not larger than $n+1$. Therefore,

$$
\prod_{k=1}^{N+1} \tilde{\rho}_{k} \geq \prod_{k=1}^{N} \rho_{k} \cdot \delta_{n+1}
$$

Lemma 3.2. For each $N$ and $q=2^{m}-1<N$, we have

$$
\left|\xi_{N}(f)\right| \leq(16 / 7)^{N} \mid\|f\|_{q} \prod_{k=q+1}^{N} \rho_{k}^{-1} .
$$

Proof. As in (17) from [5],

$$
\left|\xi_{N}(f)\right| \leq 2^{N-q}\left|\|f \mid\|_{q}\left(\Pi\left(J_{0}\right)\right)^{-1}\right.
$$

where $\Pi\left(J_{0}\right)=\min _{1 \leq j \leq N+1-q} \Pi(J)$ for $\Pi(J)$ defined in Lemma 2.3, so it is enough to estimate from below $\prod_{k=q+2}^{N+1} d_{k}(z, \tilde{Z})$ uniformly for $z \in \tilde{Z}$.

Arguing as in the proof of (2.8), we see that for each $x \in K(\gamma)$

$$
\left|e_{N+1}(x)\right|=\prod_{k=1}^{N+1} d_{k}(x, \tilde{Z}) \geq d_{1}(x, \tilde{Z}) \cdot(7 / 8)^{N} \prod_{k=2}^{N+1} \tilde{\rho}_{k}
$$


Similarly, $\prod_{k=q+2}^{N+1} d_{k}(x, \tilde{Z}) \geq(7 / 8)^{N-q-1} \prod_{k=q+2}^{N+1} \tilde{\rho}_{k}$, due to the correspondence between $d_{k}(x, \tilde{Z})$ and $\tilde{\rho}_{k}$ for $1 \leq k \leq N+1$. Removing $q+1$ smallest terms from both parts of (3.3) gives $\prod_{k=q+2}^{N+1} \tilde{\rho}_{k} \geq \prod_{k=q+1}^{N} \rho_{k}$, and the lemma follows.

The following is Theorem 1 from [3] adapted to our case.

Theorem 3.3. Suppose that $\left(B_{k}\right)_{k=1}^{\infty}$ satisfies (2.14). Then the sequence $\left(e_{N}\right)_{N=0}^{\infty}$ is a Schauder basis in the space $\mathcal{E}(K(\gamma))$.

Proof. By the Dynin-Mityagin criterion (see [7, Theorem 9]), it is enough to show that for each $p$ there is $r$ such that the sequence $\left(\left\|e_{N}\right\|_{p} \cdot\left|\xi_{N}\right|_{-r}\right)_{N=0}^{\infty}$ is bounded. Here, $|\cdot|_{-r}$ is the dual norm: for $\xi \in \mathcal{E}^{\prime}(K(\gamma))$, let $|\xi|_{-r}=\sup \left\{|\xi(f)|,\|f\|_{r} \leq 1\right\}$.

We consider only $p$ of the form $p=2^{u}$. In order to apply Lemma 2.3, we have to take $q$ of the type $q=2^{k}-1$. For this reason, given arbitrary $u$, let $q=2^{v+1}-1$, where $v=v(u)$ will be specified later. Then $r=r(q)$ is defined by (3.1).

Fix $N$ with $2^{n} \leq N<2^{n+1}$. We take $N$ so large that Lemmas 3.1 and 3.2 can be applied. Then $\left|\xi_{N}\right|_{-r} \leq C(16 / 7)^{N} \prod_{k=q+1}^{N} \rho_{k}^{-1}$ for $C$ defined by (3.1), and

$$
\left\|e_{N}\right\|_{p} \cdot\left|\xi_{N}\right|_{-r} \leq \tilde{C}\left(3 C_{0}\right)^{N} N^{p} \prod_{k=2 p+1}^{q} \rho_{k} \leq \tilde{C}\left(3 C_{0}\right)^{N} N^{p} \prod_{k=2 p+1}^{2^{v}} \rho_{k},
$$

where $\tilde{C}$ does not depend on $N$. We can decrease the upper index of the product above as all $\rho_{k}$ 's do not exceed 1 .

The product $\prod_{k=2 p+1}^{2^{v}} \rho_{k}$ takes its maximal value in the case of minimal density of points of $Z$, when each basic interval of the level $n-u$ contains $p$ points from $Z$. At worst, $\rho_{1}, \ldots, \rho_{p}$ do not exceed $\delta_{n-u}$, whereas $\rho_{p+1}, \ldots, \rho_{2 p}=\delta_{n-u-1}$, $\rho_{2 p+1}, \ldots, \rho_{4 p}=\delta_{n-u-2}$, and so on. On the other hand, values $N$ close to $2^{n+1}$ will give maximal density of $Z$ on $K(\gamma)$ with $\rho_{1}, \ldots, \rho_{p} \leq \delta_{n-u+1}, \rho_{p+1}, \ldots, \rho_{2 p}=\delta_{n-u}$, and so on. Thus, $\prod_{k=2 p+1}^{2^{v}} \rho_{k} \leq \delta_{n-u-2}^{2 p} \delta_{n-u-3}^{4 p} \cdots \delta_{n-v}^{2^{v-1}}=\exp \left[-2^{n}\left(B_{n-u-2}+\cdots+\right.\right.$ $\left.B_{n-v}\right)$ ]. We claim that the right-hand side of (3.5) is bounded for a suitable $v$. It suffices to prove that $N \log \left(3 C_{0}\right)+p \log N \leq 2^{n}\left(B_{n-u-2}+\cdots+B_{n-v}\right)$. Since $N<2^{n+1}$, it is reduced to $2 \log \left(3 C_{0}\right)+(n+1) 2^{-n} p \log 2 \leq B_{n-u-2}+\cdots+B_{n-v}$, which is valid for large $n$ if we take $v=m+u+2$, where $m$ is chosen in (2.14) for $Q=2 \log \left(3 C_{0}\right)+1$.

\section{Remarks.}

1. Under the stronger assumption (2.13), the set $K(\gamma)$ has, in addition, the extension property. The second part of the proof of Theorem 5.3 from [5] (for $j=1, s=0$ ) actually shows that the sequence $\left(\left\|\tilde{e}_{N}\right\|_{p} \cdot\left|\xi_{N}\right|_{-r}\right)_{N=0}^{\infty}$ is bounded. Here, $\tilde{e}_{N}$ is a suitable extension of $e_{N}$. Since for each extension $\tilde{f}$ of $f \in \mathcal{E}(K(\gamma))$ we have $\|f\|_{p} \leq 3|\tilde{f}|_{p}$ (by means of the Lagrange form for the Taylor remainder), this proof implies also that $\left(e_{N}\right)_{N=0}^{\infty}$ is a basis provided (2.13).

2. We conjecture that using analytic properties of $P_{2^{s}}$, one can replace $C_{0}^{N}$ in Lemma 3.1 with $N^{Q}$ for some $Q$. It would be interesting to analyze whether one can replace the exponential growth of the constant in (3.4) by a polynomial growth. 


\section{LOCAL POLYNOMIAL BASES}

In general, the system $\left(e_{N}, \xi_{N}\right)_{N=0}^{\infty}$ is not a basis. Following [3], we use local interpolations to construct bases for any considered case. Suppose we are given a nondecreasing sequence of natural numbers $\left(n_{s}\right)_{s=0}^{\infty}$. Let $N_{s}=2^{n_{s}}, M_{s}^{(l)}=$ $N_{s-1} / 2+1, M_{s}^{(r)}=N_{s-1} / 2$ for $s \geq 1$ and $M_{0}=0$. Here, $(l)$ and $(r)$ mean left and right, respectively. We choose, as above, $N_{s}$ points $\left(x_{k, j, s}\right)_{k=1}^{N_{s}}$ in each $s$ th level basic interval $I_{j, s}$. Set $e_{N, 1,0}(x)=\prod_{k=1}^{N}\left(x-x_{k, 1,0}\right)=\prod_{k=1}^{N}\left(x-x_{k}\right)$ for $x \in K(\gamma)$ and $N=0,1, \ldots, N_{0}$. Given $s \geq 1$ and $j$ with $1 \leq j \leq 2^{s}$, for $M_{s}^{(a)} \leq N \leq N_{s}$ we take $e_{N, j, s}(x)=\prod_{k=1}^{N}\left(x-x_{k, j, s}\right)$ if $x \in K(\gamma) \cap I_{j, s}$ and $e_{N, j, s}=0$ on $K(\gamma)$ otherwise. Here, the superscript $a$ in $M_{s}$ is $l$ for odd $j$ and $a=r$ if $j$ is even. Thus, we interpolate a function $f$ on the interval $I_{j, s}$ up to degree $N_{s}$, whereupon we continue this process on subintervals, preserving the previous nodes of interpolation. All $x_{k, j, s}$ 's are taken from the sequence $\left(x_{k}\right)_{1}^{\infty}$.

As above, $Z=\left(x_{k, j, s}\right)_{k=1}^{N}$ and $\left(z_{k, j, s}\right)_{k=1}^{N}$ is the same set arranged in increasing order. The functionals $\xi_{N, j, s}(f)=\left[z_{1, j, s}, \ldots, z_{N+1, j, s}\right] f$ are biorthogonal to $e_{k, i, s}$ for $N, k \in\left[M_{s}^{(a)}, N_{s}\right]$ and $i, j \in\left[1,2^{s}\right]$. But, in general, the $\xi_{N, j, s}$ are not biorthogonal to $e_{k, i, q}$. For example, $\xi_{N, 1, s+1}\left(e_{N_{s}, 1, s}\right) \neq 0$ for $M_{s+1}^{(l)} \leq N \leq N_{s}$. For this reason, as in [3], we consider the functionals

$$
\eta_{N, j, s}(f)=\xi_{N, j, s}(f)-\sum_{k=N}^{N_{s-1}} \xi_{N, j, s}\left(e_{k, i, s-1}\right) \xi_{k, i, s-1}(f)
$$

for $I_{j, s} \subset I_{i, s-1}$ and $N=M_{s}^{(a)}, M_{s}^{(a)}+1, \ldots, N_{s}$. We see that the subtrahend in $\eta_{N, j, s}$ is a kind of biorthogonal projection of $\xi_{N, j, s}$ in the dual space on the subspace spanned by $\left(\xi_{k, i, s-1}\right)_{k=N}^{N_{s-1}}$. Also, let $\eta_{N, 1,0}=\xi_{N, 1,0}$ for $0 \leq N \leq N_{0}$. By Lemma 2 in [3], the system $(e, \eta):=\left(e_{N, j, s}, \eta_{N, j, s}\right)_{s=0, j=1, N=M_{s}}^{\infty,} 2^{s}, N_{s}$ is biorthogonal. Increasing $N$ by 1 means an inclusion of one more point into the interpolation set, so, if $\eta_{N, j, s}(f)=0$ for all functionals, then $f\left(x_{k}\right)=0$ for all $k$. Since the set under consideration is perfect, the functionals $\eta$ are total on $\mathcal{E}(K(\gamma))$. Provided a suitable choice of the sequence $\left(n_{s}\right)_{0}^{\infty}$, the system $(e, \eta)$ has the basis property.

As in [5], let $n_{0}=n_{1}=2$ and $n_{s}=\left\lfloor\log _{2} \log \frac{1}{\delta_{s}}\right\rfloor$ for $s \geq 2$. Then $n_{s} \leq n_{s+1}$ and

$$
\frac{1}{2} \log \frac{1}{\delta_{s}}<N_{s} \leq \log \frac{1}{\delta_{s}} \quad \text { for } s \geq 2
$$

In the next lemma, we consider any $s, I_{j, s} \subset I_{i, s-1}$ and $N, k$, as in (4.1), that is, $M_{s}^{(a)} \leq N \leq k \leq N_{s-1}$. Also, $\prod_{m=1}^{N} \rho_{m, s}$ corresponds (in the sense of the proof of (2.8)) to the product $\prod_{m=1}^{N} d_{m}\left(x_{N+1, j, s}, Z\right)$, whereas $\prod_{m=1}^{k} \rho_{m, s-1}$ does so for $\prod_{m=1}^{k} d_{m}\left(x_{k+1, i, s-1}, W\right)$, where the points $W=\left(x_{m, i, s-1}\right)_{m=1}^{k}$ are chosen by the same rule as $Z$, but in the interval $I_{i, s-1}$. Let $q<N$.

Lemma 4.1. In the above notation, $\delta_{s-1}^{k-N} \prod_{m=q+1}^{N} \rho_{m, s} \leq \prod_{m=q+1}^{k} \rho_{m, s-1}$.

Proof. We have $N_{s-1} / 2 \leq M_{s} \leq N \leq N_{s-1}$, so $2^{n} \leq N \leq 2^{n+1}$ with $n:=n_{s-1}-1$. We can omit the case $N=2^{n+1}$ with $\prod_{m=1}^{N} \rho_{m, s}=\delta_{n+s+1} \delta_{n+s} \delta_{n+s-1}^{2} \cdots \delta_{s}^{2^{n}}$, 
since then $k=N$ and $\prod_{m=1}^{k} \rho_{m, s-1}=\delta_{n+s} \delta_{n+s-1} \delta_{n+s-2}^{2} \cdots \delta_{s-1}^{2^{n}}$, so the desired inequality is evident. Therefore, $2^{n} \leq N<2^{n+1}, \rho_{m, s} \in\left\{\delta_{n+s}, \ldots, \delta_{s}\right\}$, whereas $\rho_{m, s-1} \in\left\{\delta_{n+s}, \ldots, \delta_{s-1}\right\}$ for all considered $m$.

Let $U=W \cap I_{2 i-1, s}$ and $V=W \cap I_{2 i, s}$. Let us show that $\#(U) \leq \#(Z)$ and $\#(V) \leq \#(Z)$. By that, the densities of points from $W$ on each $s$ th level subinterval of $I_{i, s-1}$ do not exceed that of $Z$ and $\rho_{m, s} \leq \rho_{m, s-1}$ for $1 \leq m \leq N$. Since the number of points of $W$ in the interval adjacent to $I_{j, s}$ is not smaller than $k-N$, the result follows.

Suppose that $I_{j, s}$ is the right subinterval of $I_{i, s-1}$; that is $j=2 i$. If $k=2 q+1$, then $\#(Z)=N \geq q+1$. Here, $\#(U)=q+1$ and $\#(V)=q$. If $k=2 q$, then $N \geq q=\#(U)=\#(V)$. Similarly, for $j=2 i-1$ and $k=2 q+1$ we have $N \geq q+1=\#(U)$ with $\#(V)=q$, whereas $k=2 q$ gives $\#(U)=\#(V)=q \leq N$.

Since $2^{n} \leq N<2^{n+1}$, the set $Z$ contains all points of the $(n+s-1)$ th level on $I_{j, s}$ and at least one point of the $(n+s)$ th level is not included in the set. Therefore, $\rho_{1, s}=\delta_{n+s}, \rho_{2, s}=\delta_{n+s-1}$ and $\rho_{3, s} \in\left\{\delta_{n+s-1}, \delta_{n+s-2}\right\}$ depending on $N$. As in Section 2, we have $\prod_{m=1}^{N} \rho_{m, s}=\prod_{r=s}^{s+n} \delta_{r}^{s_{r}(N)}$. But $\#(W \cap I) \leq \#(Z)$, where $I$ is the subinterval of the $s$ th level containing $x_{k+1, i, s-1}$. As was mentioned in Section 2, the distribution of points from $W \cap I$ is uniform or bilateral symmetric to uniform. This means that $\rho_{m, s-1} \geq \rho_{m, s}$ for $1 \leq m \leq N$ and the degrees $s_{r}(k)$ in the representation $\prod_{m=1}^{N} \rho_{m, s-1}=\prod_{r=s-1}^{s+n} \delta_{r}^{s_{r}(k)}$ do not exceed the corresponding $s_{r}(N)$, except for the value $r=s-1$ if $\#\left(W \cap I_{j, s}\right)<\#(Z)$. In addition, we have $\rho_{N+1, s-1}=\cdots=\rho_{k, s-1}=\delta_{s-1}$. This completes the proof.

We are able to give an analogue of Theorem 2 from [3].

Theorem 4.2. Let $\left(n_{s}\right)_{s=0}^{\infty}$ be chosen as above. Then the system

$$
\left(e_{N, j, s}, \eta_{N, j, s}\right)_{s=0, j=1, N=M_{s}}^{\infty,} 2^{s}, N_{s}
$$

is a Schauder basis in the space $\mathcal{E}(K(\gamma))$.

Proof. Since in the proofs of Lemmas 3.1 and 3.2 we use only (2.1), (2.3), and the properties of uniformly distributed points, the same reasoning applies to the local case. For $2^{n} \leq N<2^{n+1}$ with $N>2 p$ we have

$$
\left\|e_{N, j, s}\right\|_{p} \leq C \cdot C_{0}^{N} N^{p} \cdot \prod_{m=2 p+1}^{N} \rho_{m, s}
$$

where the values $\rho_{m, s}$ belong to the set $\left\{\delta_{n+s}, \ldots, \delta_{s}\right\}$ and correspond to the points $Z=\left(x_{m, j, s}\right)_{m=1}^{N} \subset I_{j, s}$. In the proof, we use the notation $C$ for any constant that does not depend on $N, s$, and $j$.

As in Lemma 3.2, $\left|\xi_{N, j, s}(f)\right| \leq(16 / 7)^{N}\left|\|f \mid\|_{q} \prod_{m=q+1}^{N} \rho_{m, s}^{-1}\right.$ for $N>q+1$ with $q$ of the form $2^{m}-1$. In order to estimate the subtrahend in (4.1), we use (8) from [3]:

$$
\left|\xi_{N, j, s}\left(e_{k, i, s-1}\right)\right|=\frac{\left|e_{k, i, s-1}^{(N)}(\theta)\right|}{N !} \leq\left(\begin{array}{c}
k \\
N
\end{array}\right) \ell_{i, s-1}^{k-N} \leq\left(2 C_{0}\right)^{k} \delta_{s-1}^{k-N},
$$


since for each $\theta \in I_{j, s}$ we have $\left|e_{k, i, s-1}^{(N)}(\theta)\right| \leq \frac{k !}{(k-N) !} \prod_{m=N+1}^{k} d_{m}(\theta, Z)$ and the distances $d_{m}$ do not exceed $\ell_{i, s-1}$. As above, $\left|\xi_{k, i, s-1}(f)\right| \leq(16 / 7)^{k}\left|\|f \mid\|_{q} \times\right.$ $\prod_{m=q+1}^{k} \rho_{m, s-1}^{-1}$. By Lemma 4.1, $\left|\xi_{N, j, s}\left(e_{k, i, s-1}\right)\right| \cdot\left|\xi_{k, i, s-1}(f)\right| \leq\left(32 C_{0} / 7\right)^{k}\left|\|f \mid\|_{q} \times\right.$ $\prod_{m=q+1}^{N} \rho_{m, s}^{-1}$.

There are at most $N_{s-1}$ terms of this type in the sum in (4.1). Hence, by (3.1), $\left|\eta_{N, j, s}\right|_{-r} \leq C \prod_{m=q+1}^{N} \rho_{m, s}^{-1}\left[(16 / 7)^{N}+N_{s-1}\left(32 C_{0} / 7\right)^{N_{s-1}}\right]$. The expression in brackets is less than $2 N_{s}\left(5 C_{0}\right)^{N_{s}}$. Therefore,

$$
\left\|e_{N, j, s}\right\|_{p}\left|\eta_{N, j, s}\right|_{-r} \leq C N_{s}^{p+1} C_{2}^{N_{s}} \prod_{m=2 p+1}^{q} \rho_{m, s}
$$

with $C_{2}=5 C_{0}^{2}$. Here we can use a rough bound $\rho_{m, s} \leq \delta_{s}$ that implies

$$
\left\|e_{N, j, s}\right\|_{p}\left|\eta_{N, j, s}\right|_{-r} \leq C \exp \left[(p+1) \log N_{s}+N_{s} \log C_{2}+(q-2 p) \log \delta_{s}\right],
$$

which is bounded, by (4.2), if we take $q>2 p+\log C_{2}$ and $r$ that corresponds to this $q$ in the sense of the bound (3.1).

Remark. The argument of the theorem can be applied as well to any sequence $\left(n_{s}\right)_{s=0}^{\infty}$ with $n_{s} \uparrow \infty$ (nonstrictly) and $2^{n_{s}} \leq \log \frac{1}{\delta_{s}}$. This gives a variety of bases in the space $\mathcal{E}(K(\gamma))$. The question about quasiequivalence of these bases (see [3, p. 359]) is open.

\section{Extension Operators FOR $\mathcal{E}(K(\gamma))$}

Suppose that the space $\mathcal{E}(K)$ has a topological basis $\left(e_{n}\right)_{n=1}^{\infty}$ and that $W$ is a continuous linear extension operator for this space. Then, clearly, $W(f)$ can be given by means of individual extensions $W\left(e_{n}\right)$. Conversely, if $K$ has EP, then proper extensions of basis elements will define an extension operator. This method goes back to [7] (see also [11] for the case of compact sets with nonempty interior). Here we construct the desired operator following this approach.

Theorem 5.1. Suppose that $K(\gamma)$ has the extension property. Then an extension operator can be defined by means of proper individual extensions of basis vectors.

Proof. As is shown in [5], the condition (2.12) is a characterization of EP for $K(\gamma)$, so we suppose that $(2.12)$ is valid. We aim to show that the operator

$$
\begin{aligned}
W: \mathcal{E}(K(\gamma)) & \rightarrow C^{\infty}(\mathbb{R}): \\
f=\sum_{s=0}^{\infty} \sum_{j=1}^{2^{s}} \sum_{N=M_{s}}^{N_{s}} \eta_{N, j, s}(f) e_{N, j, s} & \mapsto \sum_{s=0}^{\infty} \sum_{j=1}^{2^{s}} \sum_{N=M_{s}}^{N_{s}} \eta_{N, j, s}(f) \tilde{e}_{N, j, s}
\end{aligned}
$$

is bounded, provided there is an appropriate choice of extensions for basis elements given in Theorem 4.2. As in [5], given $s, j$, and $N$ with $2^{n} \leq N<2^{n+1}$, we take a $C^{\infty}$-function $u(x)=u\left(x, \delta_{s+n}, I_{j, s} \cap K(\gamma)\right)$ with $\sup _{x \in \mathbb{R}}\left|u^{(p)}(x)\right| \leq c_{p} \delta_{s+n}^{-p}$, where $c_{p} \nearrow, u \equiv 1$ on $I_{j, s} \cap K(\gamma)$, and $u(x)=0$ if the distance from $x$ to the set $I_{j, s} \cap K(\gamma)$ is larger than $\delta_{s+n}$. Now we consider $e_{N, j, s}$ as a polynomial on the whole line and define $\tilde{e}_{N, j, s}$ as $e_{N, j, s} \cdot u$. 
It suffices to show that for each $p \in \mathbb{N}$ there exists $r$ such that for each $f \in$ $\mathcal{E}(K(\gamma))$ and $x \in \mathbb{R}$, we have the bound

$$
\sum_{s=0}^{\infty} \sum_{j=1}^{2^{s}} \sum_{N=M_{s}}^{N_{s}}\left|\eta_{N, j, s}(f)\right| \cdot\left|\tilde{e}_{N, j, s}^{(p)}(x)\right| \leq A_{p}\|f\|_{r},
$$

where $A_{p}$ depends only on $p$. Given $p$, let us take $M=\log C_{2}+p+3$ (with $C_{2}=5 C_{0}^{2}$ ) and $m$ that corresponds to $M$ in the sense of (2.12). Next, we take $q=2^{m+2}-1$ and the corresponding $r$ from (3.1).

By Lemma 5.2 in [5], $\left|\tilde{e}_{N, j, s}^{(p)}(x)\right| \leq C \delta_{s+n}^{-p+1} N^{p} \prod_{k=2}^{N} d_{k}(x, Z)$. As above, $C$ stands for any constant that does not depend on $s, j$, and $N$. As in Lemma 3.1, we have $d_{k}(x, Z) \leq C_{0} \rho_{k, s}$, so $\left|\tilde{e}_{N, j, s}\right|_{p} \leq C C_{0}^{N} \delta_{s+n}^{-p+1} N^{p} \prod_{k=2}^{N} \rho_{k, s}$, where the $\rho_{k, s}$ 's are defined after (4.2). By the proof of Theorem 4.2,

$$
\left|\eta_{N, j, s}(f)\right| \leq 2 N_{s} C_{2}^{N_{s}}\left|\|f \mid\|_{q} \prod_{k=q+1}^{N} \rho_{k, s}^{-1} .\right.
$$

Because of the choice of parameters, the function $\tilde{e}_{N, j, s}$ vanishes on all intervals $I_{i, s}$ for $i \neq j$. Hence, for each fixed $x$, we get at most one nonzero term in the sum with respect to $j$ in (5.1). The sum with respect to $N$ contains at most $N_{s}$ terms. Thus it remains to show that $s^{2} N_{s}^{p+2} C_{2}^{N_{s}} \prod_{k=2}^{q} \rho_{k, s}$ is bounded. As in Theorem 3.3, we replace the upper index $q$ in the product by the more convenient index $2^{m+1}$.

The product $\prod_{k=1}^{2^{m+1}} \rho_{k, s}$ takes its maximal possible value when the density of points $\left(x_{k, j, s}\right)_{k=1}^{N}$ is minimal, that is, in the case $N=M_{s}=2^{n}$ with $n:=n_{s-1}-1$. Then $\prod_{k=2}^{2^{m+1}} \rho_{k, s}=\delta_{s+n-1} \delta_{s+n-2}^{2} \cdots \delta_{s+n-m-1}^{2^{m}}$. By (2.12) and the choice of $m$, this does not exceed $\delta_{s+n-1}^{M}$ for large enough $s$. Also we have $n \geq 2$ and $\delta_{s+n-1} \leq \delta_{s} \leq$ $e^{-N_{s}}$, by (4.2). Therefore, it is enough to show that $s^{2} N_{s}^{p+2} C_{2}^{N_{s}} e^{-M N_{s}}$ is bounded, which is valid due to the choice of $M$ and the inequality $s^{2} \delta_{s}<1$.

The condition (2.14) means that the set $K(\gamma)$ is so small that the space $\mathcal{E}(K(\gamma))$ possesses a Faber basis. On the other hand, (2.14) is compatible with (2.12), which characterizes EP. We consider now the case when both conditions are valid, that is, (2.13) is satisfied. Thus we deal with an extension operator $W_{\mathrm{gl}}$ which corresponds to global interpolations of functions, that is, with the operator $W$ from the previous theorem for $s=0, j=1$. On the other hand, $W_{\mathrm{gl}}$ is equal to the accumulation part of the operator (11) from [5]. Therefore, $W_{\mathrm{gl}}$ coincides with the Pawłucki-Pleśniak operator, provided $\left(x_{k}\right)_{k=1}^{N}$ is the Fekete set. Following the proof of Theorem 5.1, we take $u(x)=u\left(x, \delta_{n}, K(\gamma)\right), \tilde{e}_{N}=e_{N} \cdot u$ and

$$
W_{\mathrm{gl}}: \mathcal{E}(K(\gamma)) \rightarrow C^{\infty}(\mathbb{R}): f=\sum_{N=0}^{\infty} \xi_{N}(f) e_{N} \mapsto \sum_{N=0}^{\infty} \xi_{N}(f) \tilde{e}_{N}
$$

Clearly, the sum $\sum_{N=0}^{M} \xi_{N} e_{N}$ is the interpolating operator $L_{M}$ for the set $Z=$ $\left(x_{k}\right)_{k=1}^{M+1}$. The parameter $\delta_{n}$ of $u$ corresponds to the values $2^{n} \leq N<2^{n+1}$. This 
gives the representation

$$
W_{\mathrm{gl}}(f, x)=f(0) \cdot u\left(x, \delta_{0}\right)+\sum_{n=0}^{\infty} \sum_{N=2^{n}}^{2^{n+1}-1}\left(L_{N}-L_{N-1}\right)(f, x) \cdot u\left(x, \delta_{n}\right),
$$

as $L_{0}(f, x)=f(0)$. After cancellation of equal terms, we get

$W_{\mathrm{gl}}(f, x)=\lim _{k \rightarrow \infty}\left\{\sum_{n=1}^{k} L_{2^{n-1}}(f, x) \cdot\left[u\left(x, \delta_{n-1}\right)-u\left(x, \delta_{n}\right)\right]+L_{2^{k+1}-1}(f, x) \cdot u\left(x, \delta_{k}\right)\right\}$.

Lemma 5.2. If (2.12) is valid, then there are $A$ and $n_{0}$ such that $\delta_{n-1}^{A} \leq \delta_{n}$ for $n \geq n_{0}$.

Proof. We use (2.12) for $M=2: \delta_{n-m}^{2^{m}} \cdots \delta_{n-1}^{2} \leq \delta_{n}$ for $n \geq n_{0}$. Clearly, the left-hand side here is larger than $\delta_{n-1}^{2^{m}}$, so we can take $A=2^{m}$ for $m=m(2)$.

Theorem 5.3. The extension operator $W_{\mathrm{gl}}$ is bounded provided (2.13).

Proof. We use the representation (5.2). Here, $\left(L_{N}-L_{N-1}\right)(f, x) \cdot u\left(x, \delta_{n}\right)=$ $A_{N, 1,0}(f, x)$ in the notation of [5]. For each $p$, we want to find $r$ with $\left|A_{N, 1,0}^{(p)}(f, x)\right| \leq$ $N^{-2}\|f\|_{r}$. Given $p$, we take $q=2^{m+1}-1$, where $m$ will be defined later, and $r=r(q)$ will be given by (3.1).

By (18) in [5], $\left|A_{N, 1,0}^{(p)}(f, x)\right| \leq C\left|\|f \mid\|_{q} \delta_{n}^{-p} N^{p}\left(2 C_{1}\right)^{N} \prod_{k=2}^{q+1} \rho_{k}\right.$. The product $\prod_{k=2}^{q+1} \rho_{k}$ can be handled as in Theorem 5.1. We are reduced to proving that the expression $\delta_{n}^{-p} N^{p+2}\left(2 C_{1}\right)^{N} \delta_{n-1} \delta_{n-2}^{2} \cdots \delta_{n-m-1}^{2^{m}}$ is bounded. Since (2.13) is stronger than (2.12), we can apply Lemma 5.2. Hence, $\delta_{n}^{-p}<\delta_{n-1}^{-p A}=\exp \left(2^{n} p A B_{n-1}\right)$. As before, $\delta_{n-1} \delta_{n-2}^{2} \cdots \delta_{n-m-1}^{2^{m}}$ is equal to $\exp \left[-2^{n}\left(B_{n-1}+\cdots+B_{n-m-1}\right)\right]$. The problem now reduces to establishing that

$$
2^{n} p A B_{n-1}+(p+2) \log N+N \log \left(2 C_{1}\right)<2^{n}\left(B_{n-1}+\cdots+B_{n-m-1}\right),
$$

which is valid for large $n$ if we define $m$ by (2.13) for $M=p A$ and $Q=$ $2 \log \left(2 C_{1}\right)+1$, as $N<2^{n+1}$.

Acknowledgment. Goncharov and Ural's work was partially supported by Tübitak grant 115F199.

\section{REFERENCES}

1. L. Frerick, Extension operators for spaces of infinite differentiable Whitney jets, J. Reine Angew. Math. 602 (2007), 123-154. Zbl 1124.46014. MR2300454. DOI 10.1515/ CRELLE.2007.005. 56

2. L. Frerick, E. Jordá, and J. Wengenroth, Tame linear extension operators for smooth Whitney functions, J. Funct. Anal. 261 (2011), no. 3, 591-603. Zbl 1232.46023. MR2799572. DOI 10.1016/j.jfa.2011.04.008. 56

3. A. Goncharov, Bases in the spaces of $C^{\infty}$-functions on Cantor-type sets, Constr. Approx. 23 (2006), no. 3, 351-360. Zbl 1112.46026. MR2201471. DOI 10.1007/s00365-005-0598-5. $56,57,63,65,66,67,68$

4. A. Goncharov, Weakly equilibrium Cantor-type sets, Potential Anal. 40 (2014), no. 2, 143-161. Zbl 1283.31001. MR3152159. DOI 10.1007/s11118-013-9344-y. 56, 57 
5. A. Goncharov and Z. Ural, Mityagin's extension problem: Progress report, J. Math. Anal. Appl. 448 (2017), no. 1, 357-375. Zbl 06663500. MR3579889. DOI 10.1016/ j.jmaa.2016.11.001. 56, 57, 62, 63, 64, 65, 66, 68, 69, 70

6. B. Malgrange, Ideals of Differentiable Functions, Tata Inst. Fund. Res. Stud. Math. 3, Oxford University Press, London, 1967. Zbl 0177.17902. MR0212575. 63

7. B. S. Mityagin, Approximate dimension and bases in nuclear spaces (in Russian), Uspekhi Mat. Nauk 16 (1961), no. 4, 63-132; English translation in Russian Math. Surveys 16 (1961), 59-128. Zbl 0104.08601. MR0152865. 65, 68

8. W. Pawłucki and W. Pleśniak, Extension of $C^{\infty}$ functions from sets with polynomial cusps, Studia Math. 88 (1988), no. 3, 279-287. Zbl 0778.26010. MR0932017. 56

9. W. Pleśniak, Markov's inequality and the existence of an extension operator for $C^{\infty}$ functions, J. Approx. Theory 61 (1990), no. 1, 106-117. Zbl 0702.41023. MR1047152. DOI 10.1016/0021-9045(90)90027-N. 56

10. M. Tidten, Fortsetzungen von $C^{\infty}$-Funktionen, welche auf einer abgeschlossenen Menge in $\boldsymbol{R}^{n}$ definiert sind, Manuscripta Math. 27 (1979), no. 3, 291-312. Zbl 0412.46027. MR0531143. DOI 10.1007/BF01309013. 56

11. D. Vogt, "Sequence space representations of spaces of test functions and distributions" in Functional Analysis, Holomorphy, and Approximation Theory (Rio de Janeiro, 1979), Lect. Notes Pure Appl. Math. 83, Dekker, New York, 1983, 405-443. Zbl 0519.46044. MR0688001. 68

12. H. Whitney, Analytic extensions of differentiable functions defined in closed sets, Trans. Amer. Math. Soc. 36 (1934), no. 1, 63-89. Zbl 0008.24902. MR1501735. DOI 10.2307/ 1989708. 63

Department of Mathematics, Bilkent University, 06800, Ankara, Turkey.

E-mail address: goncha@fen.bilkent.edu.tr; zeliha.ural@bilkent.edu.tr 